\title{
TU/e emonownen

\section{A search for natural hydrophobic deep eutectic solvents based on natural components}

\section{Citation for published version (APA):}

van Osch, D. J. G. P., Dietz, C. H. J. T., van Spronsen, J., Kroon, M. C., Gallucci, F., van Sint Annaland, M., \& Tuinier, R. (2019). A search for natural hydrophobic deep eutectic solvents based on natural components. ACS Sustainable Chemistry \& Engineering, 7(3), 2933-2942. https://doi.org/10.1021/acssuschemeng.8b03520

DOI:

10.1021/acssuschemeng.8b03520

Document status and date:

Published: 04/02/2019

\section{Document Version:}

Publisher's PDF, also known as Version of Record (includes final page, issue and volume numbers)

\section{Please check the document version of this publication:}

- A submitted manuscript is the version of the article upon submission and before peer-review. There can be important differences between the submitted version and the official published version of record. People interested in the research are advised to contact the author for the final version of the publication, or visit the $\mathrm{DOI}$ to the publisher's website.

- The final author version and the galley proof are versions of the publication after peer review.

- The final published version features the final layout of the paper including the volume, issue and page numbers.

Link to publication

\section{General rights}

Copyright and moral rights for the publications made accessible in the public portal are retained by the authors and/or other copyright owners and it is a condition of accessing publications that users recognise and abide by the legal requirements associated with these rights.

- Users may download and print one copy of any publication from the public portal for the purpose of private study or research.

- You may not further distribute the material or use it for any profit-making activity or commercial gain

- You may freely distribute the URL identifying the publication in the public portal.

If the publication is distributed under the terms of Article 25fa of the Dutch Copyright Act, indicated by the "Taverne" license above, please follow below link for the End User Agreement:

www.tue.nl/taverne

Take down policy

If you believe that this document breaches copyright please contact us at:

openaccess@tue.nl

providing details and we will investigate your claim. 


\title{
Suistalinable

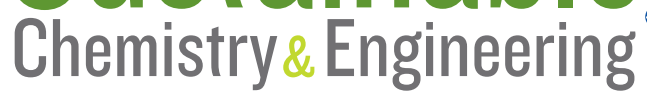 \\ A Search for Natural Hydrophobic Deep Eutectic Solvents Based on Natural Components
}

\author{
Dannie J. G. P. van Osch, ${ }^{\dagger, \S}$ Carin H. J. T. Dietz, ${ }^{\ddagger} \S$ Jaap van Spronsen, ${ }^{\dagger}$ Maaike C. Kroon, ${ }^{*}, I_{\circledast}$ \\ Fausto Gallucci, ${ }^{\ddagger}$ Martin van Sint Annaland, ${ }^{\ddagger}$ and Remco Tuinier* ${ }^{* \dagger}$ \\ ${ }^{\dagger}$ Laboratory of Physical Chemistry, Department of Chemical Engineering and Chemistry \& Institute for Complex Molecular \\ Systems, Eindhoven University of Technology, P.O. Box 513, 5600 MB Eindhoven, The Netherlands \\ ${ }^{\ddagger}$ Chemical Process Intensification, Department of Chemical Engineering and Chemistry, Eindhoven University of Technology, P.O. \\ Box 513, 5600 MB Eindhoven, The Netherlands \\ ${ }^{\mathbb{I}}$ Chemical Engineering Department, Petroleum Institute, Khalifa University of Science and Technology, P.O. Box 2533, Abu Dhabi, \\ United Arab Emirates
}

\section{Supporting Information}

ABSTRACT: Deep eutectic solvents (DESs) based on terpenes are identified and characterized. 507 combinations of solid components are tested, which results in the identification of 17 new hydrophobic DESs. Four criteria are introduced to assess the sustainability of these hydrophobic DESs from a chemical engineering point of view. These criteria include a viscosity smaller than $100 \mathrm{mPa} \cdot \mathrm{s}$, a density difference between DES and water of at least $50 \mathrm{~kg} \cdot \mathrm{m}^{-3}$ upon mixing of the DES and water, low transfer of the DES to the water phase and minor to no $\mathrm{pH}$ change. The results show that five new hydrophobic DESs based on natural components satisfy these criteria; thymol and coumarin (2:1), thymol and menthol (1:1), thymol and coumarin (1:1), thymol and menthol (1:2) and 1-tetradecanol and menthol (1:2), and thus are promising DESs. These new DESs can be considered as natural deep eutectic solvents, which have the potential to be environmentally friendly. A selected group of the hydrophobic DESs were used for the extraction of riboflavin from water. They all show higher removal of riboflavin in comparison to decanoic acid:tetraoctylammonium bromide $(2: 1)$. The highest extraction efficiency of riboflavin from water, $81.1 \%$, was achieved with the hydrophobic DES DecA:Lid (2:1).

KEYWORDS: Hydrophobic DESs, DES properties, DES leaching, Riboflavin extraction, Viscosity, Density

\section{INTRODUCTION}

In the near future, conventional solvents should be replaced by designer solvents to obey the 12 principles of Green Chemistry, introduced by Anastas and Warner. ${ }^{1}$ In 2003 a class of designer solvents, called deep eutectic solvents (DESs), were reported that could obey these principles of Green Chemistry. The first DESs reported in the literature were composed of combinations of amides and choline chloride. ${ }^{2}$ DESs consist of two or more components that liquify upon contact, which most likely is caused by entropy of mixing, hydrogen bonding and van der Waals interactions. ${ }^{3,4}$ These physical interactions are supposed to induce a dramatic decrease in the melting temperature of the mixture, as opposed to the melting temperature of the pure components, by stabilizing the liquid configuration, inducing a liquid phase at room temperature.

DES research initially focused on hydrophilic DESs. In 2015 hydrophobic DESs were reported in the literature for the first time. ${ }^{5,6}$ These were tested for the extraction of volatile fatty acids (VFAs) and biomolecules, such as caffeine and vanillin, from an aquatic environment. ${ }^{5,6}$ Although the field of hydrophobic DESs is new, already quite some papers about

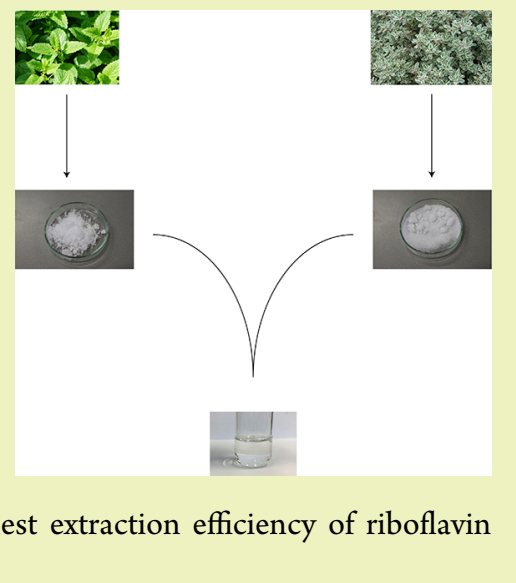


Table 1. Water Content, Density and Viscosity for Each Hydrophobic DES after Preparation ${ }^{a}$

\begin{tabular}{|c|c|c|c|}
\hline DES & Water content $[\mathrm{ppm}]$ & $\rho\left[\mathrm{kg} \cdot \mathrm{m}^{-3}\right]$ & $\eta[\mathrm{mPa} \cdot \mathrm{s}]$ \\
\hline DecA:Lid (2:1) & $253.8 \pm 3.6$ & $961.30 \pm 0.005$ & $360.60 \pm 0.10$ \\
\hline DecA:Atr (2:1) & $596.3 \pm 14.2$ & $1026.50 \pm 0.00$ & $5985.0 \pm 7.00$ \\
\hline DecA:Men (1:1) & $223.3 \pm 7.1$ & $899.77 \pm 0.030$ & $20.03 \pm 0.04$ \\
\hline DecA:Men (1:2) & $278.6 \pm 2.3$ & $899.51 \pm 0.010$ & $27.67 \pm 0.01$ \\
\hline DodE:Lid (2:1) & $151.7 \pm 2.1$ & $949.50 \pm 0.005$ & $370.55 \pm 0.15$ \\
\hline DodE:Atr (2:1) & $478.3 \pm 7.4$ & $1008.75 \pm 0.005$ & $5599.5 \pm 116.50$ \\
\hline Men:Lid (2:1) & $265.7 \pm 2.6$ & $939.18 \pm 0.005$ & $68.05 \pm 0.08$ \\
\hline Thy:Lid (2:1) & $255.1 \pm 3.2$ & $989.08 \pm 0.005$ & $122.05 \pm 0.05$ \\
\hline Thy:Cou (2:1) & $233.0 \pm 0.9$ & $1050.47 \pm 0.005$ & $31.35 \pm 0.01$ \\
\hline Thy:Men (1:1) & $306.8 \pm 4.8$ & $936.56 \pm 0.005$ & $53.14 \pm 0.00$ \\
\hline Thy:Lid (1:1) & $296.0 \pm 2.5$ & $993.12 \pm 0.005$ & $177.15 \pm 0.15$ \\
\hline Thy:Cou (1:1) & $217.3 \pm 6.3$ & $1091.80 \pm 0.005$ & $29.16 \pm 0.03$ \\
\hline Thy:Men (1:2) & $313.6 \pm 4.4$ & $923.84 \pm 0.005$ & $67.85 \pm 0.03$ \\
\hline 1-tdc:Men (1:2) & $257.9 \pm 2.9$ & $872.06 \pm 0.005$ & $43.86 \pm 0.01$ \\
\hline 1,2-dcd:Thy (1:2) & $350.9 \pm 5.5$ & $952.33 \pm 0.005$ & $64.25 \pm 0.06$ \\
\hline 1-Nap:Men (1:2) & $200.0 \pm 8.2$ & $971.10 \pm 0.005$ & $120.90 \pm 0.20$ \\
\hline Atr:Thy (1:2) & $1105.6 \pm 13.2$ & $1062.29 \pm 0.005$ & $86800^{b}$ \\
\hline
\end{tabular}

${ }^{a}$ The water contents were measured at room temperature $(295.15 \pm 1 \mathrm{~K})$, while the densities and viscosities are measured at $298 \mathrm{~K}$. All measurements were performed at atmospheric pressure $(1.01 \pm 0.03 \mathrm{bar})$. For the water contents three or four consecutive measurements were performed, while for the densities and viscosites duplicates were measured from the same batch. ${ }^{b}$ Measured only once at a shear rate of $43.4 \mathrm{~s}^{-1}$.

DESs based on natural components are generally accepted as environmentally friendly. ${ }^{21,22}$

Another improvement that we would like to introduce is testing the sustainability of these solvents from a chemical engineering point of view. If a hydrophobic solvent is too viscous or the density difference with water is too small phase separation will be difficult, which results in high energy demands. For ease of processability, the viscosity should be as low as possible, while the difference of the density between the DES and water should be as large as possible because a density difference enhances the macroscopic phase separation process to a large degree. The values were set to a viscosity smaller than $100 \mathrm{mPa} \cdot \mathrm{s}$ and a density difference between DES and water of at least $50 \mathrm{~kg} \cdot \mathrm{m}^{-3}$ upon mixing of the DES and water. Furthermore, for extractions from the water phase criteria that we aim at are a low cross contamination of the DES and water phases when used for extraction and minor to no change in the $\mathrm{pH}$. These criteria were determined via measuring the $\mathrm{pH}$, the total organic carbon (TOC) in the water phase and performing NMR analysis on the water phase. Next to the main criteria discussed before mixing, water contents are measured and thermogravimetric analysis (TGA) was performed. Nuclear magnetic resonance (NMR) of the DES is performed to investigate whether the components of the DES did not react chemically.

Here, an investigation on the search for and characterization of new hydrophobic DESs based on natural components is presented. A well classified class of natural compounds, terpenes, were selected for this research to investigate their ability to form eutectic liquid mixtures. Recently, terpenes showed great promise for the formation of eutectics. ${ }^{27}$ The combination of these components can be considered as natural deep eutectic solvents (NADES), which are generally accepted as environmentally friendly, ${ }^{21,22}$ despite further investigation into their toxicity and ecotoxicity should be performed. The following components were used as DES constituents in this work: decanoic acid (DecA), dodecanoic acid (DodE), menthol (Men), thymol (Thy), 1-tetradecanol (1-tdc), 1,2decanediol (1,2-dcd), 1-10-decanediol (1,10-dcd), cholesterol
(Chol), trans-1,2-cyclohexanediol (1,2-chd), 1-napthol (1Nap), atropine (Atr), tyramine (Tyr), tryptamine (tryp), lidocaine (Lid), cyclohexanecarboxyaldehyde (Chcd), caffeine (Caf) and coumarin (Cou). Some components were used as hydrogen bond donors (HBDs), while others were used as hydrogen bond acceptors (HBAs). A few of these components can both donate and accept hydrogen bonds. In the literature some of the combinations with lidocaine were previously presented in the literature as eutectic mixtures. ${ }^{23-25}$ More recently a debate has started on the definition of DESs, specifically on the deepness in melting point depression, and models were developed for predicting their phase diagram. ${ }^{26-32}$ Because there are still debates on the definition of DESs in the literature, for now we consider all the presented combinations as DESs.

To investigate the application potential of these hydrophobic DESs their capability to extract compounds from the water phase is studied. Riboflavin (vitamin B2) was chosen because it is an essential vitamin and it is not easy to extract from aqueous solutions. The results of the novel hydrophobic DESs presented in this study are compared to the hydrophobic DES composed of decanoic acid and tetraoctylammonium bromide in a 2:1 molar ratio, which is one of the first hydrophobic DESs presented in the literature.

\section{RESULTS AND DISCUSSION}

The tested combinations for this search are presented in Table $\mathrm{S} 1$, where the molecules expected to behave as hydrogen bond donors (HBDs) are depicted on the left side (first column) and the hydrogen bond acceptors (HBAs) on top (first row). Ratios of 2:1, 1:1 and 1:2 between the HBD and HBA were chosen and all ratios were tested on a $2 \mathrm{~g}$ scale. Components that formed no DESs are excluded. Of the 507 initial experiments, 29 mixtures liquefied upon mixing and leaving the samples at room temperature for $24 \mathrm{~h}$, which at that point were assumed to be hydrophobic DESs (green cells in Table S1).

From these expected hydrophobic DESs a batch of $50 \mathrm{~g}$ was produced, of which some of them showed some minor to 
major crystal formation on the bottom of the glass after storage for some time, up to 30 days (orange cells in Table S1). These were excluded from the screening, which resulted in a final amount of 17 hydrophobic DESs that were further investigated. These are DecA:Lid (2:1), DecA:Atr (2:1), DecA:Men (1:1), DecA:Men (1:2), DodeE:Lid (2:1), DodE:Atr (2:1), Men:Lid (2:1), Thy:Lid (2:1), Thy:Cou (2:1), Thy:Men (1:1), Thy:Lid (1:1), Thy:Cou (1:1), Thy:Men (1:2), 1-tdc:Men (1:2), 1,2-dcd:Thy (1:2), 1Nap:Men (1:2), Atr:Thy (1:2).

Densities and Viscosities. Of the 17 hydrophobic DESs selected, the water contents, densities and viscosities were measured after preparation, which are presented in Table 1.

The water contents of the DESs after preparation vary between approximately 200 to $1100 \mathrm{ppm}$, which are all low amounts of water, especially compared to the hydrophobic DESs based on quaternary ammonium salts described. ${ }^{6}$ These had water contents between 920 to $8140 \mathrm{ppm}$. Presumably, these low amounts of water in the hydrophobic DESs will only have small effects on the densities and viscosities. Of the 17 hydrophobic DESs tested, the densities of Dec:Atr (2:1), DodE:Atr (2:1), Thy:Cou (2:1), Thy:Cou (1:1), and Atr:Thy (2:1) are higher than that of water. The other 12 hydrophobic DESs have densities lower than water.

As mentioned before, a liquid viscosity that is high will lead to larger energy costs upon use in the industry. Previously, van Osch et al. ${ }^{6}$ presented DES viscosities in the range of 173 to $783 \mathrm{mPa} \cdot \mathrm{s}$, while Ribeiro et al. ${ }^{5}$ presented viscosities in the range of 10 to $220 \mathrm{mPa} \cdot \mathrm{s}$. The 17 hydrophobic DESs presented here have viscosities ranging from 20 to 86800 $\mathrm{mPa} \cdot \mathrm{s}$. Preferably, the viscosity should be as low as possible and comparable to water $(1 \mathrm{mPa} \cdot \mathrm{s})$. It is our modest opinion that viscosities up to $100 \mathrm{mPa} \cdot \mathrm{s}$ are still acceptable for industrial applications. From Table 1 it follows that the following DESs satisfy this criterion: DecA:Men (1:1), DecA:Men (1:2), Men:Lid (2:1), Thy:Cou (2:1), Thy:Men (1:1), Thy:Cou (1:1), Thy:Men (1:2), 1-tdc:Men (1:2) and 1,2-dcd:Thy (1:2).

TGA. It is useful to quantify the degradation of these hydrophobic DESs. Hence, thermograms were measured to determine the degradation temperatures of the hydrophobic DESs. They represent the weight loss of a DES as a function of temperature. Thermograms selected with the criterion of a DES viscosity lower than $100 \mathrm{mPa} \cdot \mathrm{s}$ are presented in Figure 1 and Figure 2. All spectra are presented in ESI 3.

The thermograms of the selected hydrophobic DESs all show a single-step decay of weight loss, except for Men:Lid (2:1). For this DES also a second plateau is observed. Most likely this second plateau is caused by significantly different degradation temperatures of the two components that form the DES. Moreover, it is possible that the two components only have minor interactions between them, causing two different degradation temperatures. From Figure 1 it can be seen that the difference between the hydrophobic DESs DecA:Men (1:2) and DecA:Men (1:1) is large. Despite the fact that the same components are used for these two hydrophobic DESs, the thermogram for DecA:Men (1:1) has a much more delayed decay in weight loss than DecA:Men (1:2), which is quite remarkable. It is anticipated that this effect is due to a combination of a higher volatility of Men and a more preferred liquid structure in a DecA + Men mixture at the 1:1 ratio. For the hydrophobic DESs Thy:Men (1:1) and Thy:Men (1:2)

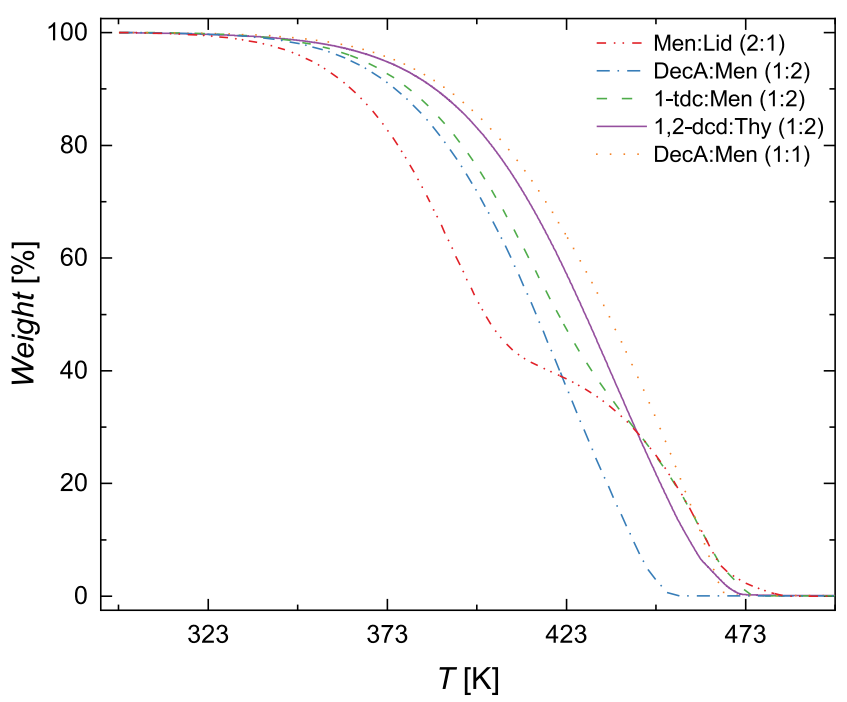

Figure 1. Thermograms of the DESs Men:Lid (2:1), DecA:Men (1:2), 1-tdc:Men (1:2), 1,2-dcd:Thy (1:2) and DecA:Men (1:1). The $x$-axis shows an increase in temperature $[\mathrm{K}]$, while the $y$-axis shows the loss in weight [\%].

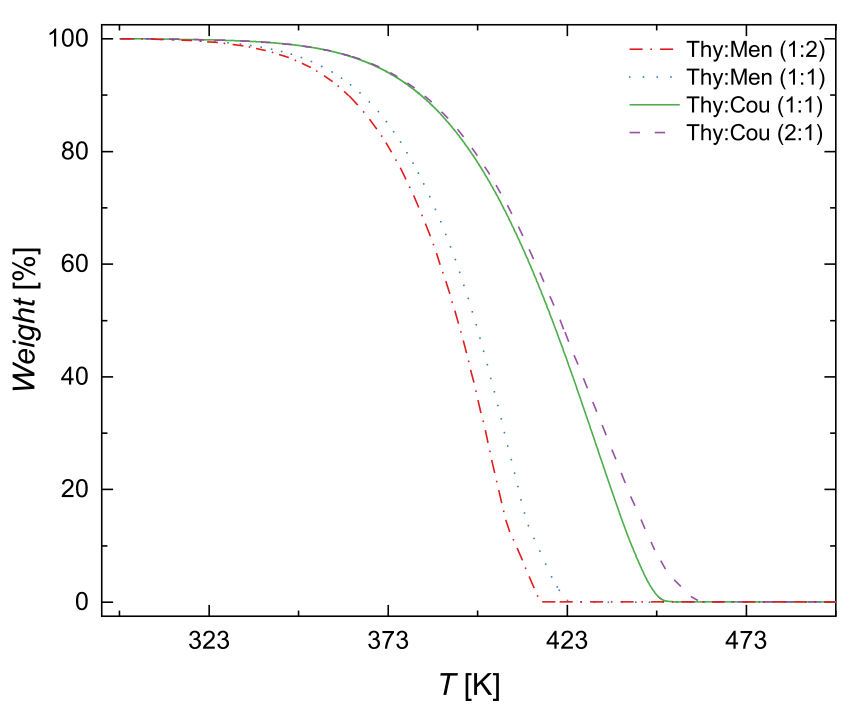

Figure 2. Thermograms of the DESs Thy:Men (1:2), Thy:Men (1:1), Thy:Cou (1:1), Thy:Cou $(2: 1)$ The $x$-axis shows an increase in temperature [K], while the $y$-axis shows the loss in weight [\%].

and for Thy:Cou (1:1) and Thy:Cou (2:1) the differences in thermograms are only small.

Table 2 gives an overview of the degradation temperatures $\left(T_{\mathrm{deg}}\right)$ from the thermograms of the hydrophobic DESs. In comparison with previously reported hydrophobic DESs based on decanoic acid and quaternary ammonium salts the $T_{\mathrm{deg}}$ values are lower. ${ }^{6}$ It is assumed that these lower $T_{\text {deg }}$ values do not present degradation, but reflect sublimation/evaporation of the components of the DESs. Components such as menthol, thymol and coumarin have a high odor and it is known that they have the ability to sublimate.

Men:Lid (2:1) has a rather low $T_{\text {deg }}$ of $363.6 \mathrm{~K}$, although it should be mentioned that this temperature is based only on the first decay and not on the second one. All hydrophobic DESs based on thymol, menthol and coumarin have a $T_{\text {deg }}$ lower than $429.6 \mathrm{~K}$. The combination of these 3 components have degradation temperatures between 378.7 and $390.8 \mathrm{~K}$. Thus, it 
Table 2. Degradation temperatures of the newly developed hydrophobic DESs

\begin{tabular}{lc} 
DES & $T_{\text {deg }}[\mathrm{K}]$ \\
\hline DecA:Lid $(2: 1)$ & $443.1 \pm 2.6$ \\
DecA:Atr $(2: 1)$ & $444.8 \pm 4.5$ \\
DecA:Men $(1: 1)$ & $410.2 \pm 2.0$ \\
DecA:Men (1:2) & $382.9 \pm 1.7$ \\
DodE:Lid $(2: 1)$ & $459.8 \pm 4.1$ \\
DodE:Atr $(2: 1)$ & $477.0 \pm 3.7$ \\
Men:Lid $(2: 1)$ & $363.6 \pm 0.4$ \\
Thy:Lid $(2: 1)$ & $412.9 \pm 4.5$ \\
Thy:Cou $(2: 1)$ & $390.8 \pm 2.9$ \\
Thy:Men (1:1) & $381.9 \pm 4.9$ \\
Thy:Lid (1:1) & $424.4 \pm 4.9$ \\
Thy:Cou (1:1) & $392.9 \pm 2.1$ \\
Thy:Men (1:2) & $378.7 \pm 3.8$ \\
1-tdc:Men (1:2) & $386.3 \pm 8.4$ \\
1,2-dcd:Thy (1:2) & $395.7 \pm 5.0$ \\
1-Nap:Men (1:2) & $388.5 \pm 6.9$ \\
Atr:Thy (1:2) & $429.6 \pm 2.1$ \\
\hline
\end{tabular}

can be observed that the combinations of the three most volatile components, thymol, menthol and coumarin, give lower $T_{\text {deg }}$ than one of these components is mixed with a less volatile component such as decanoic acid, dodecanoic acid, lidocaine or atropine, ranging from 443.1 to $477.0 \mathrm{~K}$.

These results suggest that the degradation temperature and volatility highly depend on the components that are chosen. It is an important aspect one should account for when designing a DES for a certain application and holds for both hydrophilic and hydrophobic DESs. For aprotic ionic liquids it is generally known that they all have a moderate to high degradation temperature and a low volatility, less dependent on the types of components and composition. ${ }^{33,34}$ For DESs, both the volatility as well as the degradation temperature should be properly investigated to quantify the boundary conditions when applying such solvents. Currently, in house methods are devised to obtain more knowledge regarding the volatility of DESs.

NMR on the DESs. NMR analysis was performed on the developed hydrophobic DESs. An example of an analyzed ${ }^{1} \mathrm{H}$ and ${ }^{13} \mathrm{C}$ NMR is shown in Figure 3 and Figure 4 for Thy:Cou in a molar ratio that we prepared as a 2:1 molar ratio. ${ }^{1} \mathrm{H}$ NMR

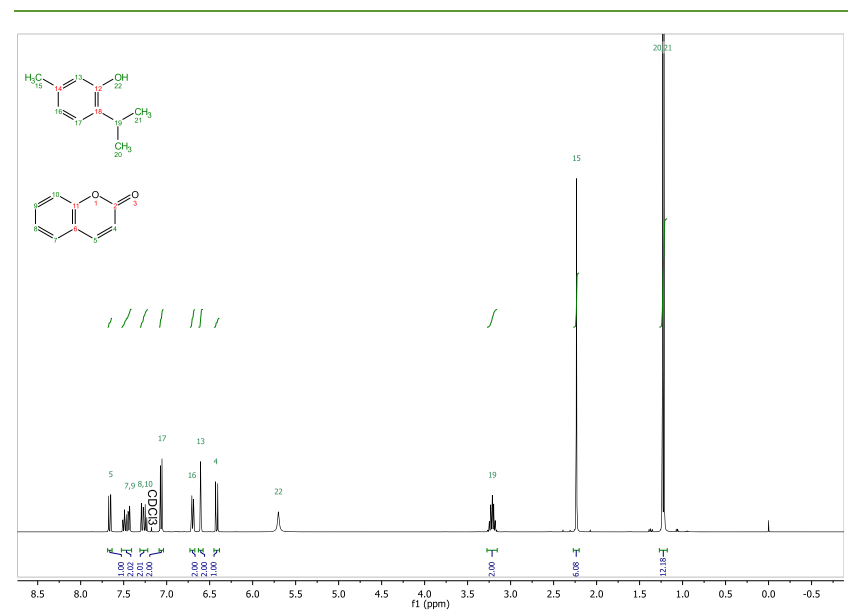

Figure 3. ${ }^{1} \mathrm{H}$ NMR of the DES Thy:Cou in a 2:1 molar ratio.

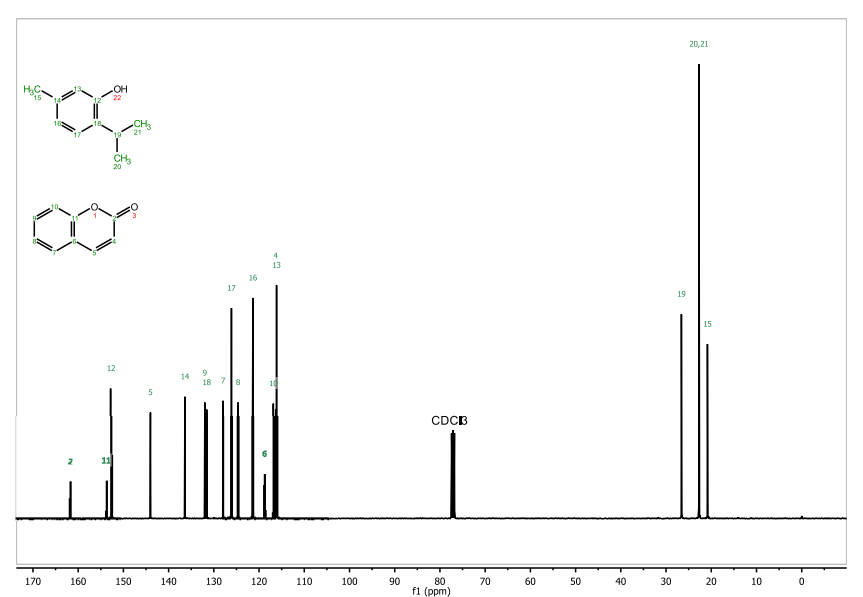

Figure $4 .{ }^{13} \mathrm{C}$ NMR of the DES Thy: Cou in a 2:1 molar ratio.

was used to verify the experimental molar ratio of the DESs and to check whether a reaction occurred between the components. The ratio of the DESs was determined by taking a specific peak of Thy and Cou and divide the integrals of these peaks. The peak of Thy at $3.25 \mathrm{ppm}$, labeled with number 19, has an integral of value 2.00 , while at $6.4 \mathrm{ppm}$, labeled with number 4 the peak of Cou has an integral value of 1.00 . Thus, the molar ratio of the Thy:Cou DES is indeed 2:1. Similar calculations were performed for the components of the other DESs and it is shown that they are all in the expected molar ratio as calculated before preparation of the DES, which is also an indication that no reaction occurred between the components.

${ }^{13} \mathrm{C}$ NMR was also performed to investigate whether a chemical reaction occurred between the two components of the DES, which would lead to extra peaks in the NMR spectrum. As can be seen in Figure 4 for the DES Thy:Cou $(2: 1)$, the peaks attributed to the original components are clearly identified and no further additional peaks were found. Similar analyses performed in all the other DESs showed that the calculations of the experimental molar ratios match the theoretical expectations, a molar ratio of $2: 1,1: 1$ and $1: 2$, which confirmed that no reactions took place between the components of the DES. All other spectra are presented in ESI 4.

Density and Viscosity after Mixing with Water. The developed hydrophobic DESs are interesting solvents for the removal or extraction of components from water, so it is useful to investigate how their properties change after mixing with water. Ideally, a fully separated system, with cross contamination as low as possible, should be achieved with a pure DES phase in contact with a pure water phase, separated by a sharp interface. First, the water contents, densities and viscosities of the DESs after mixing with water are investigated. For the hydrophobic DESs composed of DodE:Lid (2:1) and DodE:Atr (2:1), no proper phase separation could be achieved after mixing the DESs with water, which is probably due to a combination of the density that is too close to that of water, a relatively high viscosity of the DES and a DES that is less hydrophobic. The results for the other hydrophobic DESs are depicted in Table 3. Since there is a finite solubility in water, the water contents of all DESs increase after mixing with water. Typically, DESs have a low to moderate water content after mixing with water, which varies from 1.64 to $5.14 \mathrm{wt} \%$. The DES composed of 1-Nap:Men (1:2) has a rather low water 
Table 3. Water Contents, Densities and Viscosities of the Hydrophobic DESs after Mixing with Water ${ }^{a}$

\begin{tabular}{|c|c|c|c|}
\hline DES & Water content [wt \%] & $\rho\left[\mathrm{kg} \cdot \mathrm{m}^{-3}\right]$ & $\eta[\mathrm{mPa} \cdot \mathrm{s}]$ \\
\hline DecA:Lid (2:1) & $20.63 \pm 0.5$ & $983.48 \pm 0.165$ & $141.6 \pm 0.00$ \\
\hline DecA:Atr $(2: 1)$ & $33.86 \pm 0.64$ & $1025.47 \pm 0.005$ & $80.59 \pm 1.69$ \\
\hline DecA:Men (1:1) & $2.10 \pm 0.01$ & $902.94 \pm 0.005$ & $20.47 \pm 0.00$ \\
\hline DecA:Men (1:2) & $2.07 \pm 0.01$ & $902.34 \pm 0.010$ & $26.24 \pm 0.04$ \\
\hline Men:Lid (2:1) & $2.41 \pm 0.02$ & $942.19 \pm 0.00$ & $59.00 \pm 0.00$ \\
\hline Thy:Lid (2:1) & $1.64 \pm 0.01$ & $990.85 \pm 0.005$ & $100.20 \pm 0.00$ \\
\hline Thy:Cou (2:1) & $2.48 \pm 0.01$ & $1050.0 \pm 0.005$ & $26.78 \pm 0.01$ \\
\hline Thy:Men (1:1) & $1.81 \pm 0.01$ & $938.12 \pm 0.010$ & $42.01 \pm 0.02$ \\
\hline Thy:Lid (1:1) & $1.77 \pm 0.03$ & $994.37 \pm 0.005$ & $149.80 \pm 0.70$ \\
\hline Thy:Cou (1:1) & $2.54 \pm 0.02$ & $1090.24 \pm 0.005$ & $25.82 \pm 0.01$ \\
\hline Thy:Men (1:2) & $1.69 \pm 0.01$ & $925.56 \pm 0.00$ & $52.17 \pm 0.02$ \\
\hline 1-tdc:Men (1:2) & $1.73 \pm 0.01$ & $874.22 \pm 0.00$ & $36.63 \pm 0.01$ \\
\hline 1,2-dcd:Thy (1:2) & $5.14 \pm 0.02$ & $956.31 \pm 0.005$ & $42.57 \pm 0.02$ \\
\hline 1-Nap:Men (1:2) & $0.13 \pm 0.01$ & $981.94 \pm 0.005$ & $74.43 \pm 0.13$ \\
\hline Atr:Thy $(1: 2)$ & $3.00 \pm 0.05$ & $1061.02 \pm 0.110$ & $9255.5 \pm 264$ \\
\hline
\end{tabular}

${ }^{a_{T}}$ The water contents of the water saturated DES were measured at room temperature $(295.15 \pm 1 \mathrm{~K})$, while the densities and viscosities were measured at $298 \mathrm{~K}$. All measurements were performed at atmospheric pressure $(1.01 \pm 0.03 \mathrm{bar})$. For the water contents three or four consecutive measurements were performed, while for the densities and viscosites duplicates were measured from the same batch.

content, of only $0.13 \mathrm{wt} \%$. Two of the developed DESs have a high water content. DecA:Lid $(2: 1)$ has a water content of 20.6 wt \%, while this is 33.9 wt \% for DecA:Atr (2:1). It is hypothesized that this is attributed to the their less hydrophobicity and the fact that they easily form complexes with water.

The densities change slightly due to the uptake of water. The largest changes occur for DecA:Lid (2:1) and 1-Nap:Men (1:2). When these hydrophobic DESs are used in combination with water, complete phase separation is a necessity. In theory, phase separation gets more difficult (slower) when the density of the hydrophobic DES is closer to the density of water. The densities of DecA:Lid (2:1), DecA:Atr (2:1), Thy:Lid (2:1) and Thy:Lid (1:1) are very close to the density of water, which can delay phase separation and enhance emulsification. Preferably, the density between the DES and water is as large as possible, at least $50 \mathrm{~kg} \cdot \mathrm{m}^{-3}$ and larger, such as for the DESs DecA:Men (1:1), DecA:Men (1:2), Men:Lid (2:1), Thy:Cou (2:1), Thy:Men (1:1), Thy:Cou (1:1), Thy:Men (1:2) and 1-tdc:Men (1:2). Regarding the fact if the density is higher or lower than water has in our opinion no influence on its use. Most likely, a density lower or higher than water can even be used in it benefit.

The addition of water to a hydrophobic DES normally leads to a decrease in viscosity, due to a small amount of water uptake combined with the fact that the viscosity of pure water is smaller. For most of the hydrophobic DESs tested here this applies. A small increase in viscosity is however observed for the DES DecA:Men (1:1). A thorough explanation for this behavior lacks, but one of the possibilities is that water is to some degree emulsified. For most DESs there is only a tiny decrease of the viscosity. Apparently, the increase of water content from ppm levels to approximately 1.64 to $5.14 \mathrm{wt} \%$ induces only minor changes. For example, DecA:Lid (2:1) has only a minor change in viscosity, while the increase in water content is large. The large decrease in viscosity for DecA:Atr (2:1) however does correspond to the increase of its water content. Moreover, the Atr:Thy (1:2) DES has a considerable decrease in the viscosity when water is added. To summarize, the DESs that satisfy the viscosity of $100 \mathrm{mPa} \cdot \mathrm{s}$ or lower after mixing with water are Deca:Atr (2:1), DecA:Men (1:1),
DecA:Men (1:2), Men:Lid (2:1), Thy:Cou (2:1), Thy:Men (1:1), Thy:Cou (1:1), Thy:Men (1:2), 1-tdc:Men (1:2) and 1,2-dcd:Thy (1:2) and 1-Nap:Men (1:2).

NMR after Mixing with Water. NMR analysis is also performed on the hydrophobic DESs after mixing with $\mathrm{H}_{2} \mathrm{O}$. For the DES Dode:Lid (2:1) and DodE:Atr (2:1) no suitable NMR analysis after mixing with water could be achieved due to difficulties with separating the two phases. As an example, for the DES composed of Thy and Cou the integrals in the ${ }^{1} \mathrm{H}$ NMR, Figure 5, of the peaks at 3.25 and 6.4 ppm give a molar

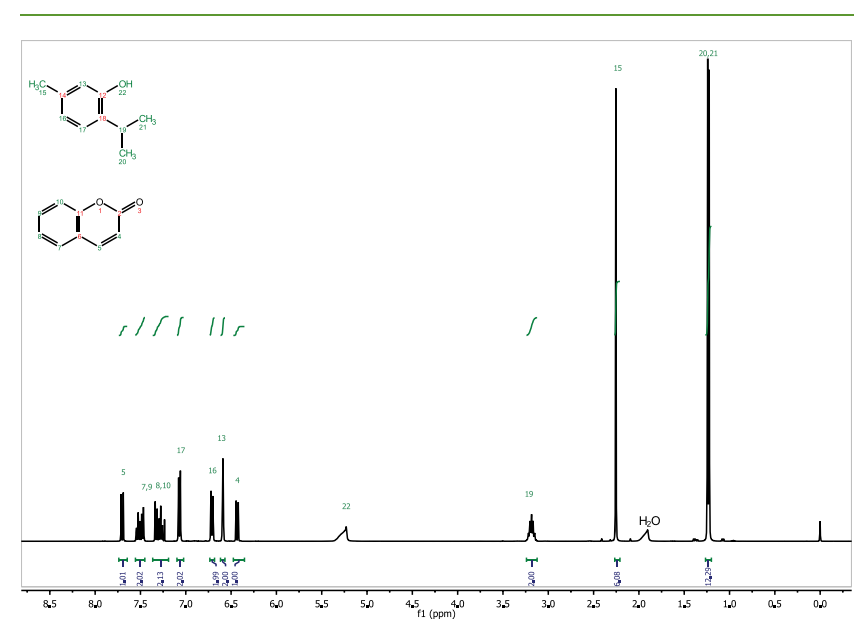

Figure 5. ${ }^{1} \mathrm{H}$ NMR of the DES Thy:Cou in a 2:1 molar ratio after mixing with $\mathrm{H}_{2} \mathrm{O}$.

ratio of 2:1 Thy:Cou, showing that the ratio of the DES is still the same. Moreover, all the other integrals are also in the expected ratio. A small peak around $1.8 \mathrm{ppm}$ is present, which is not related to the pure DES components, hence it could be attributed to a small contamination, in this case by water, that is present either in the DES phase or the solvent $\mathrm{CDCl}_{3}$.

In the ${ }^{13} \mathrm{C} N M R$, Figure 6, no extra peaks were observed, a sign that no reaction occurred between the two components of the DESs. All other NMRs of the DESs after mixing with water were analyzed in the same manner and showed molar ratios as 


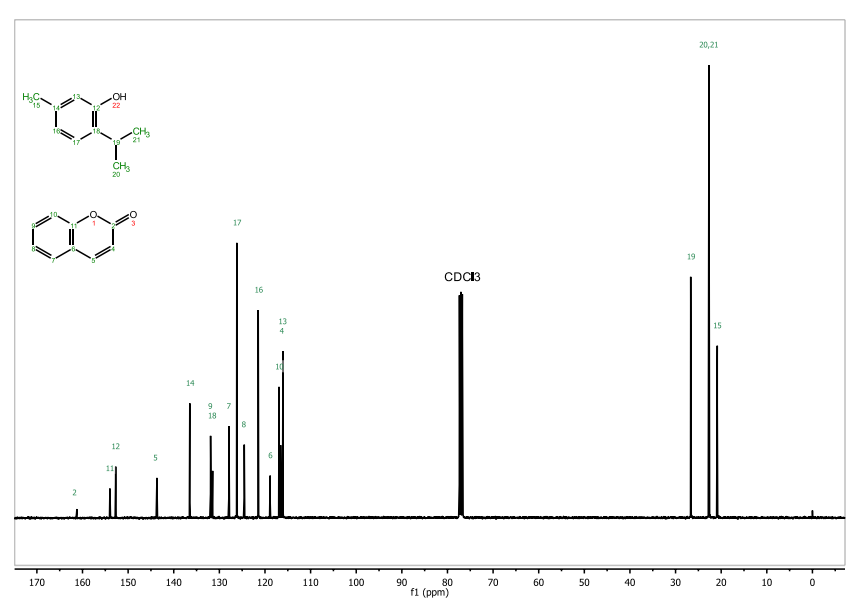

Figure $6 .{ }^{13} \mathrm{C}$ NMR of the DES Thy:Cou in a 2:1 molar ratio after mixing with $\mathrm{H}_{2} \mathrm{O}$.

expected. Thus, no reactions occurred between the components. The spectra are presented in ESI 5.

Contamination of the Water Phase by the Hydrophobic DES. An important aspect that should be investigated is the contamination of the water phase with hydrophobic DES. Ideally, the amount of DES that transfers to the water phase should be as low as possible. This contamination was investigated by measuring the $\mathrm{pH}$, the total organic carbon (TOC) content in the water phase and by performing NMR on the water phase, with a suppression of the water peak.

$\mathrm{pH}$ of the Water Phase. When applying hydrophobic DESs for extraction purposes it is useful to verify whether the $\mathrm{pH}$ of the water phase upon mixing of the two phases is affected, since the change of $\mathrm{pH}$ of the water phase can be an indication that DES transferred to the water phase. A $\mathrm{pH}$ change can have a negative result on an extraction and if microorganisms are present in the water phase, it can even lead to their destruction. Furthermore, acidification or basification of water can have an undesirable effect on equipment. Thus, as a boundary condition the $\mathrm{pH}$ of the water phase after mixing with the hydrophobic DES should be between 6 and 8. It can be expected that if one of the two components is acidic or basic, the water phase will also become more acidic of basic. From Table 4 it can be observed that eight hydrophobic DESs satisfy this criterion, namely DecA:Lid (2:1), DecA:Atr (2:1), Thy:Cou (2:1), Thy:Men (1:1), Thy:Cou (1:1), Thy:Men (1:2), 1-tdc:Men (1:2), Atr:Thy (1:2). Surprisingly, DecA:Lid (2:1) has a neutral $\mathrm{pH}$, while all water phases mixed with a DES composed of decanoic acid are acidic and all water phases mixed with a DES containing lidocaine become basic. Most likely the acidic and basic effect cancel each other. The same also applies for DecA:Atr (2:1), where decanoic acid contains a carboxylic group and atropine contains an amine group. Moreover, it is remarkable that Atr:Thy (1:2) has a $\mathrm{pH}$ of 7.53. Atropine contains an amine group, while thymol has no groups that should induce a $\mathrm{pH}$ change so upon transfer of the components to the water phase it is expected they induce a $\mathrm{pH}$ $>7$. Finally, it is peculiar that the $\mathrm{pH}$ of the water phase after contact with 1,2-dcd:Thy (1:2) is 4.64. Both components of these DES are alcohols, so the $\mathrm{pH}$ is expected to be close to 7 upon transfer to the water phase.

Amount of Hydrophobic DES Transferred to the Water Phase upon Mixing. One of the techniques used for the determination of the amount of hydrophobic DES present
Table 4. Amount of Hydrophobic DES Transferred to the Water Phase upon Mixing ${ }^{a}$

\begin{tabular}{lcrl}
\multicolumn{1}{c}{ DES } & pH [-] & $\begin{array}{c}\text { TOC } \\
\text { value } \\
{[\mathrm{ppm}]}\end{array}$ & $\begin{array}{c}\text { molar ratio between the } \\
\text { components in the water } \\
\text { phase }[-]\end{array}$ \\
DecA:Lid (2:1) & 6.96 & 58489 & - \\
DecA:Atr (2:1) & 6.54 & 6475 & - \\
DecA:Men (1:1) & 4.16 & 276 & - \\
DecA:Men (1:2) & 4.29 & 390 & - \\
Men:Lid (2:1) & 10.04 & 2108 & Men:Lid 1.0:7.1 \\
Thy:Lid (2:1) & 9.15 & 777 & Thy:Lid 1.6:1.0 \\
Thy:Cou (2:1) & 7.64 & 1162 & Thy:Cou -1.0:1.3 \\
Thy:Men (1:1) & 7.24 & 583 & Thy:Men 3.0:1.0 \\
Thy:Lid (1:1) & 9.29 & 1175 & Thy:Lid 1.0:6.0 \\
Thy:Cou (1:1) & 6.97 & 1596 & Thy:Cou 1.0:2.9 \\
Thy:Men (1:2) & 7.34 & 474 & Thy:Men 1.6:1.0 \\
1-tdc:Men (1:2) & 7.14 & 273 & 1-tdc:Men 1.0:3.2 \\
1,2-dcd:Thy (1:2) & 4.64 & 898 & 1,2-dcd:Thy 1.0:2.9 \\
1-Nap:Men (1:2) & 5.95 & 795 & 1-Nap:Men 5.3:1.0 \\
Atr:Thy (1:2) & 7.53 & 4917 & Atr:Thy 24.2:1.0 \\
DecA:N $8888-B r(2: 1)$ & 1.6 & - & -
\end{tabular}

${ }^{a_{T}}$ TOC values in the water phases measured with a Shimadzu TOC-L $\mathrm{CPH} / \mathrm{CPN}$ (see also the Experimental Section) and the molar ratios of the components present in the water phase.

in the water phase is TOC. The TOC content was measured to quantify the amount of dissolved organic molecules in the water phase (Table 4). The TOC value of DecA:Lid $(2: 1)$ is considerably higher than the amounts measured previously. ${ }^{7}$ This can be explained by the difficult sampling of the water phase after centrifugation. All DESs prepared with atropine led to high TOCs in the coexisting water phase. The water phases in contact with hydrophobic DESs prepared with menthol had TOC values lower than $0.1 \mathrm{wt} \%$, except for Men:Lid (2:1). It is hypothesized that this is caused by a complexation of menthol with lidocaine, leading to a complex that becomes slightly more hydrophilic in comparison with its pure component. Remarkably, also the DESs composed of thymol and coumarin have slightly higher TOC values. Overall, it can be concluded that the amount of organics that transfer to the water phase is rather low in comparison to the previously reported hydrophobic DESs. ${ }^{6,7}$

The amount of the organic phase that transfers to the water phase should be as low as possible. Previous research on the quaternary ammonium salt based hydrophobic DESs showed that the lowest amount of organics in the water phase was $1.9 \%$, which was caused by the quaternary ammonium salt tetraoctylammonium bromide. ${ }^{6}$ Other research performed NMR on the water phase, without suppression of the water peak, and showed that components such as acetic acid and tetrabutylammonium chloride leach to the water phase. ${ }^{35}$

${ }^{1} \mathrm{H}$ NMR analysis was performed with a suppression of the water peak to determine the ratio of the components of the hydrophobic DES that transfer to the water phase. An example is given in Figure 7 for the water phase after mixing it with the hydrophobic DES Thy:Men (1:1). The specific peaks for Thy were located between 6.6 and $7.2 \mathrm{ppm}$, while Men had peaks just before $2.0 \mathrm{ppm}$ and between 3.0 and $3.5 \mathrm{ppm}$. For example, the integral of the peak of Thy at $7.2 \mathrm{ppm}$ can be divided by the integral of the peak of Men at $3.4 \mathrm{ppm}$. This gives a molar ratio of Thy:Men in the water phase of 3.0:1.0. The spectrum also shows that within the same molecule there is a difference in the estimated integrals, as is shown for Thy 


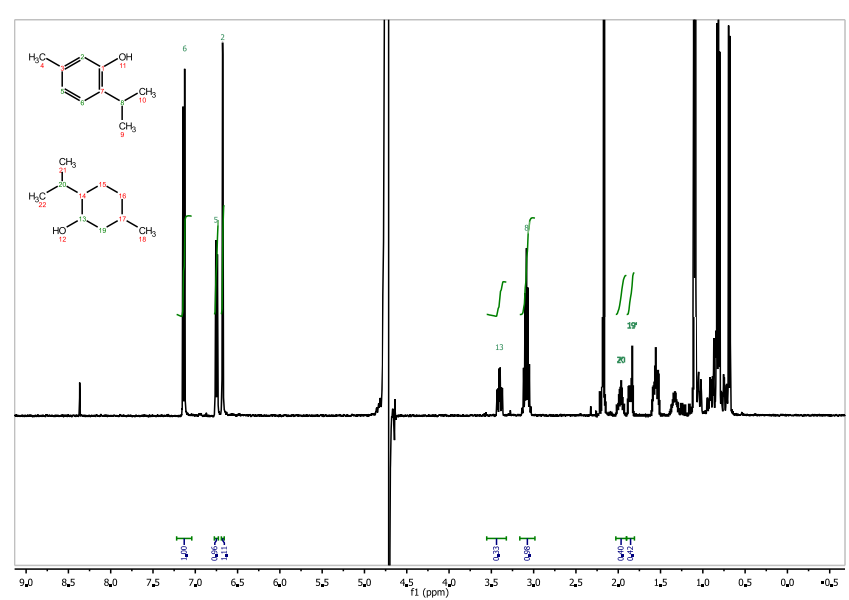

Figure 7. ${ }^{1} \mathrm{H}$ NMR of the water phase after mixing with Thy:Men (1:1) with a suppression of the water peak.

between 6.6 and $7.2 \mathrm{ppm}$. This could be caused by impurities present in the original components of the DESs that at these low concentrations may still be visible.

Despite the fact that this method gives no precise ratios of which component transfers to the water phase, it gives a good indication of the different molar ratios of the components in the water phase. The estimated ratios in water are given in Table 4. It is clear that the portioning of the two DES components over the DES-rich and water-rich phases is rather asymmetric and random. Further detailed analyses or interpretation of the current data is not viable, neither within the scope of the current work, but certainly worth exploring in future works. All spectra are presented in ESI 6.

Application: Extraction of Riboflavin from Water. To verify whether the selected hydrophobic DESs are capable of extracting compounds from water, the removal of rifoblavin (vitamin B2) from the aqueous solutions is investigated. Riboflavin was chosen because it is one of the most necessary vitamins for humans and challenging to extract from aqueous solutions. High performance liquid chromatography (HPLC) was used to measure the concentration of riboflavin before and after extraction. From the concentrations the extraction efficiencies $(E E)$ can be calculated:

$$
E E[\%]=\frac{C_{\mathrm{vitB2}}^{\mathrm{ini}}-C_{\mathrm{vitB} 2}^{\mathrm{fin}}}{C_{\mathrm{vitB} 2}^{\mathrm{ini}}} \times 100 \%
$$

In eq $1, C_{\text {vitB2 }}^{\text {ini }}$ is the initial concentration of the vitamin $\mathrm{B}$ in $\mathrm{H}_{2} \mathrm{O}$ in wt $\%$ and $C_{\text {vitB2 }}^{\text {fin }}$ is the final concentration of vitamin $B$ in $\mathrm{H}_{2} \mathrm{O}$ in wt \% after mixing with the DES. This implies that complete extraction corresponds to $E E=100 \%$ and no extraction implies $E E=0 \%$. EE values between 30 and $70 \%$ can be seen a moderate, while $>70 \%$ can be seen a good EE. The results are presented in Table 5.

The results of the selected hydrophobic DESs as presented in Table 5 were compared with one of the first developed ones, the DES composed of decanoic acid and tetraoctylammonium bromide in a $2: 1$ ratio. $^{6}$ This DES had a EE of $19.0 \%$, while DecA:Men (1:1) extracted the lowest amount of the new hydrophobic DESs with approximately a $E E$ of $20.5 \%$. Thus, all new hydrophobic DESs had a higher extraction efficiency than the one composed of decanoic acid and tetraoctylammonium bromide. The highest extraction efficiency was achieved with the hydrophobic DES DecA:Lid (2:1), a DES which is also
Table 5. DES-Water Distribution Coefficients $(E E)$ of Riboflavin for $\mathrm{H}_{2} \mathrm{O}$ for the Different Selected Hydrophobic DESs

\begin{tabular}{lc}
\multicolumn{1}{c}{ DES } & $E E[\%]$ \\
\hline DecA:Men $(1: 1)$ & $20.5 \pm 1.5$ \\
DecA:Lid $(2: 1)$ & $81.1 \pm 4.4$ \\
1-tdc:Men (1:2) & $61.1 \pm 0.6$ \\
Thy:Cou $(2: 1)$ & $70.6 \pm 0.6$ \\
Thy:Cou $(1: 1)$ & $47.5 \pm 0.3$ \\
Thy:Men (1:2) & $53.6 \pm 0.2$ \\
Thy:Men $(1: 1)$ & $54.3 \pm 0.0$ \\
DecA: $\mathrm{N}_{8888} \mathrm{Br}$ & $19.0 \pm 1.2$ \\
\hline
\end{tabular}

very capable of extracting metal ions from water. ${ }^{7}$ Varying the ratios of the DES had no influence on the distribution coefficients for the DES consisting of thymol and menthol, while difference in extraction efficiency of approximately $23.1 \%$ was observed for the DES that was prepared from thymol and coumarin. In general, all newly developed hydrophobic DESs showed moderate to good distribution coefficients. The precise reasons for this is not clear, but it is hypothesized that different factors play a role in this, e.g., $\mathrm{pH}$ change of the water phase due to certain solubilities of the pure components, the hydrophobicity of the DES and interaction between the DES and riboflavin.

Conclusions and Outlook. In this work a series of new, hydrophobic DESs based on natural components were reported. From 507 combinations of two solid components, 17 became a liquid at room temperature, which were further assessed for their sustainability via four criteria. These criteria are based on the use of the hydrophobic DESs as extractants and include a viscosity below $100 \mathrm{mPa} \cdot \mathrm{s}$, a density that should be rather different than the density of the water phase $(50 \mathrm{~kg}$. $\mathrm{m}^{-3}$ ) a limited $\mathrm{pH}$ change of the water phase upon mixing with water and a low amount of DES that transfers to the water phase.

More than 10 DESs follow the viscosity criterion below 100 $\mathrm{mPa} \cdot \mathrm{s}$. Regarding the density, the criterion was set at a density difference between the DES and water as large as possible $(\rho \geq$ $\left.50 \mathrm{~kg} \cdot \mathrm{m}^{-3}\right)$.

The hydrophobic DESs Deca:Men (1:1), DecA:Men (1:2), Men:Lid (2:1), Thy:Cou (2:1), Thy:Men (1:1), Thy:Cou (1:1), Thy:Men (1:2) and 1-tdc:Men (1:2) satisfy this criterion.

Furthermore, the criterion of a limited $\mathrm{pH}$ change (between 6 and 8) of the water phase coexisting with the DES showed that the hydrophobic DESs DecA:Lid (2:1), DecA:Atr (2:1), Thy:Cou (2:1), Thy:Men (1:1), Thy:Cou (1:1), Thy:Men (1:2), 1-tdc:Men (1:2) and Atr:Thy (1:2) have a negligible $\mathrm{pH}$ change. The amount of organics that transfers to the water phase was comparable for all developed hydrophobic DESs, except for DecA:Lid (2:1), DecA:Atr (2:1) and Atr:Thy (1:2), which had considerably higher TOC values.

In general, the newly developed DESs Thy:Cou (2:1), Thy:Men (1:1), Thy:Cou (1:1), Thy:Men (1:2) and 1tdc:Men (1:2) satisfied all four criteria. Therefore, these hydrophobic DESs may be considered as relatively sustainable, hydrophobic designer solvents. These DESs were used for the removal of riboflavin from an aqueous environment. All new hydrophobic DESs showed moderate to high extraction yields. The highest extraction efficiency of riboflavin, $81.1 \%$, was achieved with the hydrophobic DES DecA:Lid (2:1). 


\section{EXPERIMENTAL SECTION}

Materials and Preparation. Materials. All chemicals were ordered from Sigma-Aldrich, expect for riboflavin which was purchased from TCI Chemicals. Their purities (as stated by the supplier) and CAS numbers are depicted in Table 6.

Table 6. Components Used in This Investigation and Their Purities $^{a}$

\begin{tabular}{llc}
\multicolumn{1}{c}{ component } & CAS & purity [wt \%] \\
decanoic acid (DecA) & $334-48-5$ & $\geq 98$ \\
dodecanoic acid (DodE) & $143-07-7$ & $\geq 99$ \\
menthol (Men) & $89-78-1$ & $\geq 99$ \\
thymol (Thy) & $89-83-8$ & $\geq 99$ \\
1-tetradecanol (1-tdc) & $112-72-1$ & $\geq 97$ \\
1,2-decanediol (1,2-dcd) & $1119-86-4$ & $\geq 98$ \\
1,10-decanediol (1,10-dcd) & $112-47-0$ & $\geq 98$ \\
cholesterol (chol) & $57-88-5$ & $\geq 92.5$ \\
trans-1,2-cyclohexanediol (1,2-chd) & $460-57-7$ & $\geq 98$ \\
1-napthol (1-Nap) & $90-15-3$ & $\geq 99$ \\
atropine (Atr) & $51-55-8$ & $\geq 99$ \\
tyramine (Tyr) & $51-67-2$ & $\geq 98$ \\
tryptamine (Tryp) & $61-54-1$ & $\geq 98$ \\
lidocaine (Lid) & $137-58-6$ & $\geq \mathrm{N} . \mathrm{A}$. \\
cyclohexanecarboxaldehyde (Chcd) & $2043-61-0$ & $\geq 97$ \\
caffeine (Caf) & $58-08-2$ & $\geq 98$ \\
coumarin (Cou) & $91-64-6$ & $\geq 99$ \\
riboflavin & $83-88-5$ & $\geq \mathrm{N} . \mathrm{A}$. \\
As stated by the supplier. & & \\
\end{tabular}

Preparation of the Hydrophobic DESs. The formation of hydrophobic DESs was tested by mixing two solid components at three different molar ratios, e.g., 2:1, 1:1 and 1:2, with a total weight of $2 \mathrm{~g}$. The different molar ratios were chosen to verify whether DES liquids can be prepared over a wide or small compositional range. A Mettler Ax205 balance with an uncertainty of $20 \mu \mathrm{g}$ was used for weighing the chemicals. The first component was weighed directly in the flask, in which a magnetic stirrer was already present. The second component was first weighed on a weighing boat, after which the correct amount was transferred into the flask. The components in the flask were premixed on a VWR Analog Vortex Mixer and heated in a metal heating block. An IKA RCT basic was used for monitoring the stirring speed and temperature. An IKA ETS-D5 temperature controller with an uncertainty of $\pm 0.1 \mathrm{~K}$ was used for temperature control (Table 7).

The formation of the hydrophobic DESs was investigated via a standard procedure. After preparation and premixing, the flasks were heated and stirred for $2 \mathrm{~h}$ at a temperature of approximately $313 \mathrm{~K}$. In case a homogeneous liquid sample was produced, the flask was kept at room temperature for $24 \mathrm{~h}$ to check its stability. The sample was considered a DES if no crystals were visually present in the liquid after $24 \mathrm{~h}$. The samples that formed no liquid were further heated to $333.15 \mathrm{~K}$. The same procedure as explained before was used. If no liquid was formed at $333.15 \mathrm{~K}$ the temperature was heated to 353.15 $\mathrm{K}$, after which the same procedure was used.

The combinations of components that formed a DES on the 2-g scale, were scaled up to form $50 \mathrm{~g}$ of DES. For the $50 \mathrm{~g}$ scale it was investigated whether the DESs were also formed at room temperature. Only the DESs that were also stable at a 50-g scale were further analyzed. During the analyses, crystals appeared in some DESs within the time frame of a month. Those DESs were discarded from the investigation and not further analyzed.

After the preparation the DES was stored in a closed vial until all measurements were performed simultaneously.

Mixing with Water. $18.0 \mathrm{~g}$ of Milli-Q water was weighed in a 50 $\mathrm{mL}$ centrifuge tube (CELLSTAR), after which $18.0 \mathrm{~g}$ of hydrophobic DES was added. Proper mixing was induced via an IKA KS 4000 I
Table 7. Hydrogen Bond Donor (HBD), Hydrogen Bond Acceptor (HBA), Molar Ratio of between the HBD and HBA and the Abbreviation of the DES

\begin{tabular}{llcl} 
HBD & HBA & molar ratio & \multicolumn{1}{c}{ abbreviation } \\
decanoic acid & lidocaine & $2: 1$ & DecA:Lid $(2: 1)$ \\
decanoic acid & atropine & $2: 1$ & DecA:Atr $(2: 1)$ \\
decanoic acid & menthol & $1: 1$ & DecA:Men $(1: 1)$ \\
decanoic acid & menthol & $1: 2$ & DecA:Men $(1: 2)$ \\
dodecanoic acid & lidocaine & $2: 1$ & DodE:Lid $(2: 1)$ \\
dodecanoic acid & atropine & $2: 1$ & DodE:Atr $(2: 1)$ \\
menthol & lidocaine & $2: 1$ & Men:Lid $(2: 1)$ \\
thymol & lidocaine & $2: 1$ & Thy:Lid $(2: 1)$ \\
thymol & coumarin & $2: 1$ & Thy:Cou $(2: 1)$ \\
thymol & menthol & $1: 1$ & Thy:Men $(1: 1)$ \\
thymol & lidocaine & $1: 1$ & Thy:Lid $(1: 1)$ \\
thymol & coumarin & $1: 1$ & Thy:Cou $(1: 1)$ \\
thymol & menthol & $1: 2$ & Thy:Men $(1: 2)$ \\
1-tetredecanol & menthol & $1: 2$ & 1-tdc:Men $(1: 2)$ \\
1,2-decanediol & thymol & $1: 2$ & 1,2-dcd:Thy $(1: 2)$ \\
1-napthol & menthol & $1: 2$ & 1-Nap:Men $(1: 2)$ \\
atropine & thymol & $1: 2$ & Atr:Thy $(1: 2)$ \\
\hline
\end{tabular}

incubating shaker (500 rpm, RT). After shaking, the DES and water phase were separated via centrifugation with a Sigma 2-16 KL centrifuge. The DESs DecA:Men (1:1), DecA:Men (1:2), Men:Lid (2:1), Thy:Lid (2:1), Thy:Cou (2:1), Thy:Men (1:1), Thy:Lid (1:1), Thy:Cou (1:1), Thy:Men (1:2), 1-tdc:Men (1:2), 1,2-dcd:Thy (1:2) and 1-Nap:Men (1:2) were centrifuged for $10 \mathrm{~min}$ at $6000 \mathrm{rpm}$, while the DESs DecA:Lid (2:1), DecA:Atr (2:1), DodE:Lid (2:1), DodE:Atr (2:1) and Atr:Thy (1:2) were centrifuged for $60 \mathrm{~min}$ at $12,000 \mathrm{rpm}$. After centrifugation, the DES and water phase were taken with a needled syringe. For the DESs DodE:Lid (2:1) and DodE:Atr (2:1) no proper phase separation could be achieved. Thus, these were excluded from analysis after mixing with $\mathrm{H}_{2} \mathrm{O}$.

Analytical Methodologies. Water Content. The water contents of the DESs were measured with a Mettler Toledo D39 Karl Fischer titration apparatus (coulometer). The DESs DecA:Lid $(2: 1)$ and DecA:Atr (2:1) had a high water content (>10 wt \%) and could not be properly measured on the Toledo equipment. Those samples were measured on a Metrohm type 899 coulometer. The coulometers were filled with $20 \mathrm{~mL}$ chloroform and $80 \mathrm{~mL}$ Hydranal Coulomat AG. The DES 1-Nap:Men (1:2) could not be measured on a coulometric Karl Fischer, so it was measured on a volumetric Karl Fischer apparatus. A Metrohm type 795 KFT volumetric Karl Fischer was used. Before use of the Karl Fischer apparatuses, they were checked with water standards of $0.01,0.1$ and $1.0 \mathrm{wt} \%$ of water. All measurements were conducted at room temperature $(295.15 \pm 1 \mathrm{~K})$ and atmospheric pressure $(1.01 \pm 0.03 \mathrm{bar})$.

Viscosity and Density. The measured physicochemical properties of the hydrophobic DESs are the density and the viscosity. The density was determined with an Anton-Paar DMA $4500 \mathrm{M}$ with a deviation of the density of $\pm 0.05 \mathrm{~kg} \cdot \mathrm{m}^{-3}$ and a temperature variation of $\pm 0.05 \mathrm{~K}$. The discrepancy of the density from the several reference oils that were measured was not more than $\pm 0.01 \mathrm{~kg} \cdot \mathrm{m}^{-3}$. The viscosity was measured with an Anton Paar Lovis $2000 \mathrm{ME}$ rolling ball viscometer. All hydrophobic DESs, except for Atr:Thy (1:2), were measured in a glass capillary with an inner diameter of $1.8 \mathrm{~mm}$ equipped with a gold-coated ball. The coefficient of variation was below $0.2 \%$, while the forward/backward deviation was at its most $1.0 \%$. The capillary was calibrated with a N26 synthetic base oil, which was supplied by Paragon scientific ltd. For the DES Atr:Thy (1:2) the viscosity measurement before mixing was performed on an Anton Paar Physica MCR 301 rheometer, because of its high viscosity. A concentric cylinder system (CC27) was used. The inner diameter is $26.66 \mathrm{~mm}$, while the outer diameter is $28.92 \mathrm{~mm}$. Measurements and calibration were performed as presented elsewhere. ${ }^{10}$ After mixing with $\mathrm{H}_{2} \mathrm{O}$, the capillary of $2.5 \mathrm{~mm}$ was used for 
Atr:Thy (1:2), also equipped with a gold-coated ball. The variation coefficient for this capillary was below $0.5 \%$, while the forward/ backward deviation was at most $1.0 \%$. The capillary was calibrated with a N100 synthetic base oil.

The measurements of the water content, density and viscosity were performed within a time difference that was as short as possible. From the total material two syringes with samples were taken, after which one sample was used for measuring the water content while the other sample was used for measuring the density and viscosity.

Thermogravimetric Analysis (TGA). The decomposition temperatures of the DESs were measured with a TGA Q500 from TA Instruments. The weight accuracy is $0.1 \%$, while the temperature accuracy is $1 \mathrm{~K}$. A heating rate of $10 \mathrm{~K} \cdot \mathrm{min}^{-1}$ was used from 298.15 to $873.15 \mathrm{~K}$. The degradation temperature is the weight loss onset temperature determined via the step tangent method. By determining the point of contact of the tangent of the $x$-axis and the $y$-axis the temperature at which weight loss starts can be determined. The thermograms were analyzed with the TA Instruments Universal Analysis 2000 software (version 4.5A, Build 4.5.0.5).

Nuclear Magnetic Resonance (NMR). The goal of the NMR analyses was investing whether reactions occur between the components of the DES. Both proton $\left({ }^{1} \mathrm{H}\right)$ and carbon $\left({ }^{13} \mathrm{C}\right)$ nuclear magnetic resonance (NMR) were performed. A Bruker 400 automatic NMR was used with 128 scans and a relaxation time of $3 \mathrm{~s}$ for the ${ }^{1} \mathrm{H}$ and 1000 scans with a relaxation time of $3 \mathrm{~s}$ for the ${ }^{13} \mathrm{C}$. $8^{\prime \prime}$ Wilmad NMR tubes with an outside diameter of $5 \mathrm{~mm}$ were used for the measurements, in which DES diluted with chloroform was added. Analysis of the spectra was conducted with Mestrenova (version v11.0.4-18998) and ChemBiodraw (version 348-208690-1653).

For the analysis of the ${ }^{1} \mathrm{H}$ NMRs it was checked whether all peaks of the protons were in the theoretical molar ratio to investigate whether no reactions between the components of the DES occurred. Therefore, the integral of a specific peak of the molecule used as HBD was compared to the integral of a specific peak for the molecule used as HBA. For the ${ }^{13} \mathrm{C}$ NMRs it was checked whether extra peaks were present, which is an indication of reactions between the two components of the DES.

If the molar ratios are correct in the ${ }^{1} \mathrm{H}$ NMR spectrum and no extra peaks appear in the ${ }^{13} \mathrm{C}$ NMR spectrum it can be concluded that no reactions between the two components of the DES occurred.

$\mathrm{pH}$ of the Water Whase after Mixing the DESs with Water. The $\mathrm{pH}$ of the water phase was measured with a Mettler Toledo seven compact $\mathrm{pH} /$ ion meter (S220). A 5 point calibration was performed in the range of a $\mathrm{pH}$ of 2 to 7 . Standards with higher $\mathrm{pH}$, up to a $\mathrm{pH}$ of 10 , were tested for calibration and also gave adequate results. The $\mathrm{pH}$ measuring range of the meter is from -2 to +20 . The $\mathrm{pH}$ accuracy is \pm 0.002 , while the accuracy of the temperature is $\pm 0.5 \mathrm{~K}$. The probe connected to the $\mathrm{pH}$ meter is an InLab Micro 51343160.

Total Organic Content (TOC). The total organic carbon (TOC) of the water phase was determined by a Shimadzu TOC-L CPH/CPN with auto sampler ASI-L ( $24 \mathrm{~mL}$ vails). The amount of TOC was calculated by two calibrations curves $(0-10 \mathrm{ppm}$ and $0-100 \mathrm{ppm})$. The vial was filled with a 100 times diluted sample and 0.5 wt \% 1 $\mathrm{mol} \cdot \mathrm{L}^{-1}$ hydrochloric acid was automatically added before injection. The injection volume was set to $50 \mu \mathrm{L}$. The TOC amount was measured in duplicate with an uncertainty $<1.5 \%$.

NMR of the Water Phase. The molar ratio between the two components of the hydrophobic DES that transferred to the water phase was investigated with ${ }^{1} \mathrm{H}$ NMR. After mixing water with the DES, a sample of the water phase was taken and added to a WilmadLabGlass 528-pp-7 tube containing a coaxial insert (WilmadLabGlass WGS-5BL) with $\mathrm{D}_{2} \mathrm{O}$. The measurements were performed with a Bruker 400 automatic NMR and the water peak was suppressed, by using the software program Bruker NMR. A relaxation time of $3 \mathrm{~s}$ was used and 100 scans were performed. This adjustment allowed analyzing the spectra of the low concentrated DESs in the water phase, as follows. The ${ }^{1} \mathrm{H}$ NMR spectra showed the protons of the components of the hydropobic DES present in the water phase. Well-defined peaks of the HBD and HBA were integrated. By dividing the integral of specific peaks obtained for the HBD and HBA component, the molar ratio at which the two components of the DES were present in the water phase could be estimated. The localization of the DES components peaks in the water phase was based on the NMRs of the pure components in $\mathrm{CDCl}_{3}$. The results showed a standard deviation within the same molecule of approximately $10 \%$.

\section{ASSOCIATED CONTENT}

\section{S Supporting Information}

The Supporting Information is available free of charge on the ACS Publications website at DOI: 10.1021/acssuschemeng.8b03520.

TGAs of all prepared hydropobobic DESs and NMRs of the developed DESs before and after mixing with water and the water phases after mixing with water (PDF)

\section{AUTHOR INFORMATION}

\section{Corresponding Authors}

*R. Tuinier. E-mail: r.tuinier@tue.nl. Phone: +31 402477345.

*M. C. Kroon. E-mail: maaike.kroon@ku.ac.ae. Phone: +971 2 6075317.

ORCID $\odot$

Maaike C. Kroon: 0000-0002-5985-986X

Remco Tuinier: 0000-0002-4096-7107

Author Contributions

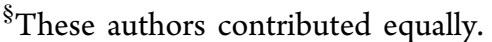

Notes

The authors declare no competing financial interest.

\section{ACKNOWLEDGMENTS}

The authors thank Dr. A. Catarina C. Esteves for the help with the NMR study and a critical reading of the manuscript. Furthermore, The authors would like to thank the members of the ISPT "Deep Eutectic Solvents in the pulp and paper industry" consortium for their financial and in kind contribution. This cluster consists of the following organisations: Altri-Celbi, Buckman, Crown Van Gelder, CTP, DS Smith Paper, ESKA, Essity, Holmen, ISPT, Mayr-Melnhof Eerbeek, Metsä Fibre, Mid Sweden University, Mondi, Omya, Parenco BV, The Navigator Company, Sappi, Essity, Smurfit Kappa, Stora Enso, Eindhoven University of Technology, University of Aveiro, University of Twente, UPM, Valmet Technologies Oy, Voith Paper, VTT Technical Research Centre of Finland Ltd, WEPA and Zellstoff Pöls. Furthermore, this project received funding from the Bio-Based Industries Joint Undertaking under the European Union's Horizon 2020 research and innovation programme under grant agreement Provides No. 668970. This work was performed under the framework of Chemelot InSciTe-Horizontal project and with contributions from the European Regional Development Fund (ERDF) within the framework of OP-Zuid and with contributions from the province of Brabant and Limburg and the Dutch Ministry of Economic Affairs.

\section{REFERENCES}

(1) Anastas, P. T.; Warner, J. C. Green Chemistry: Theory and Practice; Oxford University Press, 1998.

(2) Abbott, A.; Capper, G.; Davies, D.; Rasheed, R.; Tambyrajah, V. Novel solvent properties of choline chloride/urea mixtures. Chem. Commun. 2003, 70-71.

(3) Smith, E. L.; Abbott, A. P.; Ryder, K. S. Deep Eutectic Solvents (DESs) and Their Applications. Chem. Rev. 2014, 114, 11060-11082. 
(4) Francisco, M.; van den Bruinhorst, A.; Kroon, M. C. LowTransition-Temperature Mixtures (LTTMs): a New Generation of Designer Solvents. Angew. Chem., Int. Ed. 2013, 52, 3074-3085.

(5) Ribeiro, B. D.; Florindo, C.; Iff, L. C.; Coelho, M. A.; Marrucho, I. M. Menthol-Based Eutectic Mixtures: Hydrophobic Low Viscosity Solvents. ACS Sustainable Chem. Eng. 2015, 3, 2469-2477.

(6) van Osch, D. J. G. P.; Zubeir, L. F.; van den Bruinhorst, A.; Rocha, M. A.; Kroon, M. C. Hydrophobic Deep Eutectic Solvents as Water-Immiscible Extractants. Green Chem. 2015, 17, 4518-4521.

(7) van Osch, D. J. G. P.; Parmentier, D.; Dietz, C. H. J. T.; van den Bruinhorst, A.; Tuinier, R.; Kroon, M. C. Removal of alkali and transition metal ions from water with hydrophobic deep eutectic solvents. Chem. Commun. 2016, 52, 11987-11990.

(8) Tereshatov, E.; Boltoeva, M. Y.; Folden, C. First Evidence of Metal Transfer into Hydrophobic Deep Eutectic and Low-TransitionTemperature Mixtures: Indium Extraction from Hydrochloric and Oxalic Acids. Green Chem. 2016, 18, 4616-4622.

(9) Schaeffer, N.; Martins, M. A.; Neves, C. M.; Pinho, S. P.; Coutinho, J. A. Sustainable hydrophobic terpene-based eutectic solvents for the extraction and separation of metals. Chem. Commun. 2018, 54, 8104-8107.

(10) Dietz, C. H.; Kroon, M. C.; Di Stefano, M.; van Sint Annaland, M.; Gallucci, F. Selective separation of furfural and hydroxymethylfurfural from an aqueous solution using a supported hydrophobic deep eutectic solvent liquid membrane. Faraday Discuss. 2018, 206, 77-92.

(11) Florindo, C.; Oliveira, F. S.; Rebelo, L. P. N.; Fernandes, A. M.; Marrucho, I. M. Insights into the Synthesis and Properties of Deep Eutectic Solvents Based on Cholinium Chloride and Carboxylic Acids. ACS Sustainable Chem. Eng. 2014, 2, 2416-2425.

(12) Dietz, C. H. J. T.; van Osch, D. J. G. P.; Kroon, M. C.; Sadowski, G.; van Sint Annaland, M.; Gallucci, F.; Zubeir, L. F.; Held, C. PC-SAFT Modeling of CO2 Solubilities in Hydrophobic Deep Eutectic Solvents. Fluid Phase Equilib. 2017, 448, 94-98.

(13) Zubeir, L. F.; Van Osch, D. J. G. P.; Rocha, M. A. A.; Banat, F.; Kroon, M. C. Carbon dioxide solubilities in decanoic acid-based hydrophobic deep eutectic solvents. J. Chem. Eng. Data 2018, 63, 913-919.

(14) Arain, M. B.; Yilmaz, E.; Soylak, M. Deep eutectic solvent based ultrasonic assisted liquid phase microextraction for the FAAS determination of cobalt. J. Mol. Liq. 2016, 224, 538-543.

(15) Zhu, S.; Zhou, J.; Jia, H.; Zhang, H. Liquid-liquid microextraction of synthetic pigments in beverages using a hydrophobic deep eutectic solvent. Food Chem. 2018, 243, 351-356.

(16) Cao, J.; Yang, M.; Cao, F.; Wang, J.; Su, E. Well-designed hydrophobic deep eutectic solvents as green and efficient media for the extraction of artemisinin from artemisia annua leaves. ACS Sustainable Chem. Eng. 2017, 5, 3270-3278.

(17) de Faria, E. L.; do Carmo, R. S.; Cláudio, A. F. M.; Freire, C. S.; Freire, M. G.; Silvestre, A. J. Deep Eutectic Solvents as Efficient Media for the Extraction and Recovery of Cynaropicrin from Cynara cardunculus L. Leaves. Int. J. Mol. Sci. 2017, 18, 2276.

(18) Hayyan, M.; Hashim, M. A.; Al-Saadi, M. A.; Hayyan, A.; AlNashef, I. M.; Mirghani, M. E. S. Assessment of cytotoxicity and toxicity for phosphonium-based deep eutectic solvents. Chemosphere 2013, 93, 455-459.

(19) Wen, Q.; Chen, J.-X.; Tang, Y.-L.; Wang, J.; Yang, Z. Assessing the toxicity and biodegradability of deep eutectic solvents. Chemosphere 2015, 132, 63-69.

(20) Radošević, K.; Bubalo, M. C.; Srček, V. G.; Grgas, D.; Dragičević, T. L.; Redovniković, I. R. Evaluation of toxicity and biodegradability of choline chloride based deep eutectic solvents. Ecotoxicol. Environ. Saf. 2015, 112, 46-53.

(21) Paiva, A.; Craveiro, R.; Aroso, I.; Martins, M.; Reis, R. L.; Duarte, A. R. C. Natural deep eutectic solvents-solvents for the 21st century. ACS Sustainable Chem. Eng. 2014, 2, 1063-1071.

(22) Liu, Y.; Friesen, J. B.; McAlpine, J. B.; Lankin, D. C.; Chen, S.N.; Pauli, G. F. Natural Deep Eutectic Solvents: Properties, Applications, and Perspectives. J. Nat. Prod. 2018, 81, 679-680.
(23) Corvis, Y.; Négrier, P.; Lazerges, M.; Massip, S.; Léger, J.-M.; Espeau, P. Lidocaine/L-menthol binary system: cocrystallization versus solid-state immiscibility. J. Phys. Chem. B 2010, 114, 54205426.

(24) Kang, L.; Jun, H.; McCall, J. Physicochemical studies of lidocaine-menthol binary systems for enhanced membrane transport. Int. J. Pharm. 2000, 206, 35-42.

(25) Kang, L.; Jun, H.; Mani, N. Preparation and characterization of two-phase melt sys- tems of lidocaine. Int. J. Pharm. 2001, 222, 3544.

(26) Martins, M. A.; Pinho, S. P.; Coutinho, J. A. Insights into the Nature of Eutectic and Deep Eutectic Mixtures. J. Solution Chem. 2018, 1-21.

(27) Martins, M. A. R.; Crespo, E. A.; Pontes, P. V.; Silva, L. P.; Bülow, M.; Maximo, G. J.; Batista, E. A. C.; Held, C.; Pinho, S. P.; Coutinho, J. A. Tunable hydrophobic eutectic solvents based on terpenes and monocarboxylic acids. ACS Sustainable Chem. Eng. 2018, 6, 8836-8846.

(28) Crespo, E. A.; Silva, L. P.; Martins, M. A.; Fernandez, L.; Ortega, J.; Ferreira, O.; Sadowski, G.; Held, C.; Pinho, S. P.; Coutinho, J. A. Characterization and Modeling of the Liquid Phase of Deep Eutectic Solvents Based on Fatty Acids/Alcohols and Choline Chloride. Ind. Eng. Chem. Res. 2017, 56, 12192-12202.

(29) Crespo, E. A.; Silva, L. P.; Martins, M. A.; Bülow, M.; Ferreira, O.; Sadowski, G.; Held, C.; Pinho, S. P.; Coutinho, J. A. P. The Role of Polyfunctionality in the Formation of [Ch] Cl-Carboxylic AcidBased Deep Eutectic Solvents. Ind. Eng. Chem. Res. 2018, 57, 1119511209.

(30) Pontes, P. V. A.; Crespo, E. A.; Martins, M. A. R.; Silva, L. P.; Neves, C. M. S. S.; Maximo, G. J.; Hubinger, M. D.; Batista, E. A. C.; Pinho, S. P.; Coutinho, J. A. P.; Sadowski, G.; Held, C. Measurement and PC-SAFT modeling of solid-liquid equilibrium of deep eutectic solvents of quaternary ammonium chlorides and carboxylic acids. Fluid Phase Equilib. 2017, 448, 69-80.

(31) Silva, L. P.; Fernández, L.; Conceição, J. H.; Martins, M. A. R.; Sosa, A.; Ortega, J.; Pinho, S. P.; Coutinho, J. A. Design and characterization of sugar-based deep eutectic solvents using COSMORS. ACS Sustainable Chem. Eng. 2018, 6, 10724-10734.

(32) Kollau, L. J.; Vis, M.; van den Bruinhorst, A.; Esteves, A. C. C.; Tuinier, R. Quantification of the liquid window of deep eutectic solvents. Chem. Commun. 2018, 54, 13351-13354.

(33) Maton, C.; De Vos, N.; Stevens, C. V. Ionic liquid thermal stabilities: decomposition mechanisms and analysis tools. Chem. Soc. Rev. 2013, 42, 5963-5977.

(34) Wooster, T. J.; Johanson, K. M.; Fraser, K. J.; MacFarlane, D. R.; Scott, J. L. Thermal degradation of cyano containing ionic liquids. Green Chem. 2006, 8, 691-696.

(35) Florindo, C.; Branco, L.; Marrucho, I. Development of Hydrophobic Deep Eutectic Sol- vents for Extraction of Pesticides from Aqueous Environments. Fluid Phase Equilib. 2017, 448, 135142 . 